\title{
The Emerging Role of Telomerase Reverse Transcriptase in Mitochondrial DNA Metabolism
}

\author{
Donna M. Gordon ${ }^{1}$ and Janine Hertzog Santos $^{2}$ \\ ${ }^{1}$ Department of Biological Sciences, Mississippi State University, 114 Harned Hall, 295 Lee Boulevard, \\ Mississippi State, MS 39762, USA \\ ${ }^{2}$ Department of Pharmacology and Physiology, New Jersey Medical School of UMDNJ, 185 South Orange Avenue, \\ Medical Sciences Building, H653, Newark, NJ 07103, USA
}

Correspondence should be addressed to Janine Hertzog Santos, santosja@umdnj.edu

Received 14 May 2010; Revised 26 July 2010; Accepted 31 August 2010

Academic Editor: Ashis Basu

Copyright (C) 2010 D. M. Gordon and J. H. Santos. This is an open access article distributed under the Creative Commons Attribution License, which permits unrestricted use, distribution, and reproduction in any medium, provided the original work is properly cited.

\begin{abstract}
Telomerase is a reverse transcriptase specialized in telomere synthesis. The enzyme is primarily nuclear where it elongates telomeres but recent reports have shown that it also localizes to mitochondria. The function of TERT in mitochondria is largely unknown but the available findings point to a role in mitochondrial DNA metabolism. This paper discusses the available data on mitochondrial telomerase with particular emphasis on its effects upon the organellar DNA.
\end{abstract}

\section{Introduction}

Telomerase is a reverse transcriptase well recognized for its function at telomeres, protein-DNA structures essential for the stability of linear chromosomes. The enzyme is composed minimally of two different subunits, a catalytic core (TERT) responsible for DNA catalysis and an RNA component (TERC) that is used as template for telomeric DNA synthesis. Whereas the RNA is present in both somatic and germ cells constitutively, expression of TERT is tightly regulated. Telomerase activity is high during embryogenesis and in the vast majority of tumors but is low or non-existent in most adult somatic cells [1]. Both TERT mRNA and protein have been reported in telomerase-negative cells albeit at low levels $[2,3]$.

In recent years many different reports have indicated that the reverse transcriptase component of telomerase has additional cellular functions beyond telomere stabilization. For instance, TERT has been shown to play a role in chromatin remodeling and in DNA damage response and to act as a transcriptional modulator of the Wnt/betacatenin signaling pathways $[4,5]$. More recently it was shown to acquire properties of an RNA-dependent RNA polymerase when in a complex with the RNA component of the mitochondrial endoribonuclease MRP; such activity is not involved in the maintenance of telomeres but rather in regulation of gene expression [6].

In line with the idea that TERT has extratelomeric functions, we were the first to show that the human protein (hTERT) is also mitochondrial $[7,8]$. The mitochondrial localization of endogenous and ectopically expressed hTERT was subsequently reproduced by others [9-12]. The presence of hTERT in mitochondria was surprising given the wellestablished role of telomerase in telomere maintenance and the lack of a telomeric structure on the mitochondrial DNA (mtDNA). However, it was not entirely unexpected as cytoplasmic localization of telomerase had been previously reported [13-17] and, as described above, other functions outside telomere maintenance have been increasingly ascribed to hTERT.

This paper focuses on the localization of TERT to mitochondria and its putative role in mtDNA metabolism. We review findings regarding the effects of mitochondrial hTERT upon genotoxic stress and recent evidence demonstrating the impact of TERT (or lack thereof) to mitochondrial biology under nonstress conditions. The impact of mitochondrial telomerase to aging and telomere maintenance is not discussed as it has been reviewed 
elsewhere [18]. More information about the noncanonical and/or extratelomeric activities of telomerase can be found in other recent reviews [19-21].

\section{Mitochondrial Localization of TERT}

2.1. Endogenous and Ectopically Expressed TERT Are Mitochondrial. The first evidence that TERT was a mitochondrial protein came from studies done by us initially identifying a mitochondrial-targeting signal (MTS) at its N-terminus [7]. Using software packages that predict MTSs, we identified the first 20 amino acid residues of TERT to be a putative mitochondrial targeting peptide. We found the MTS to be conserved among higher eukaryotes such as plants, fish, and mammals but not present in lower species such as yeast and tetrahymena [7]. Fusion of EGFP to the C-terminus of full-length wild-type (WT) human TERT showed that the recombinant protein was localized to mitochondria. Addition of only the MTS to the N-terminus of EGFP rendered the fluorescent protein completely mitochondrial and mutations of two amino acid residues within the MTS abolished hTERT's mitochondrial localization, indicating that the MTS was sufficient and required to target hTERT to mitochondria $[7,8]$. Telomerase enzymatic activity was also detected in purified mitochondrial extracts from various human cells [7], collectively providing strong evidence that hTERT was a mitochondrial protein. Subsequently, others have confirmed the presence of endogenous hTERT in mitochondria by immunofluorescence and Western blotting [912]. Of note, a recent study showed that hTERT is found in purified mitochondria of primary umbilical vein endothelial cells [12], underscoring that the mitochondrial localization of hTERT is not an artifact of protein overexpression or associated with cellular transformation. In this context, our unpublished work shows that a fraction of endogenous rat and mouse TERT is mitochondrial as well (Green et al. in preparation).

The content of hTERT in cancer cells has been estimated to range from 20 to 50 molecules [22]. According to recent reports $[11,12]$ and to our unpublished data, we estimate that $\sim 10-20 \%$ of the total cellular hTERT is mitochondrial under nonstress conditions, which is equivalent to 3-10 molecules of hTERT/mitochondrion at least in cancer cells. It is not surprising then that many have missed the mitochondrial localization of TERT. The unequal subcellular distribution of dually targeted proteins is a wide spread phenomenon and has been termed "eclipsed distribution" [23]. Many of these proteins have been shown to have distinct functions in the different subcellular compartments [23-26] similar to what is predicted for TERT activity.

2.2. Submitochondrial Localization of TERT. To gain insight into the molecular function of a mitochondrial protein it is necessary to define its submitochondrial localization since its presence in the mitochondrial membrane (inner or outer) or in the matrix would predict different biological functions. Haendeler et al. [11] identified the submitochondrial localization of hTERT by immunoblots and coimmunoprecipitations. The authors isolated mitochondria from Hek293, treated the purified organelles with proteinase $\mathrm{K}$, and probed for hTERT by Western blots. Using this approach they concluded that hTERT was mostly localized to the mitochondrial matrix. Their claims were substantiated by experiments demonstrating that overexpressed myc-tagged hTERT co-immunoprecipitated with TOM20, TOM40, and TIM23 [11]. TOM20, TOM40, and TIM23 are protein translocases of the outer (TOM20 and TOM40) and inner (TIM23) mitochondrial import machinery [24]. These results are consistent with experiments showing that hTERT binds mtDNA (see below), which resides in the matrix.

\section{Regulation of hTERT's Subcellular Distribution}

It is now recognized that the dual targeting of proteins can be regulated, induced, or rebalanced in response to cellular signaling or to changes in extracellular conditions [26]. While much is still unknown about how the subcellular distribution of TERT is achieved, the available data suggest that it is tightly regulated. In this section, we review published information regarding the signals that target TERT to the nucleus and mitochondria and the role of oxidative stress in redistributing hTERT within the cell. An excellent review about mechanisms, regulation, and function of dual targeting of proteins has been recently published [26].

3.1. TERT's Targeting Signals. It is clear that at basal levels TERT is primarily nuclear with only a small fraction present in mitochondria. However, it is currently unknown what regulates the intracellular distribution of TERT. In addition to its N-terminal MTS, hTERT has a nuclear export signal (NES) spanning amino acids 978-987 [14, 27]. Several nuclear localization signals (NLSs) are predicted but they have not yet been mapped out onto the protein [14]. No splicing variants involving the MTS or the NES of hTERT have been reported neither have variants that change its subcellular distribution been identified. It therefore seems likely that the dual targeting of TERT is based on a single translation product containing different targeting signals. In such a case, the balance between the mitochondrial and nuclear pools of the protein may be determined by various competing factors, including the affinity of each signal for its target or, alternatively, the accessibility of the target signal for specific receptor binding [26].

3.2. Regulation of TERT's Distribution by Oxidative Stress. The intracellular distribution of hTERT changes upon specific stimuli, and many different proteins have been associated with its shuttling between nucleus and cytoplasm (including mitochondria) $[10,12,14,16,28-32]$. Under oxidative stress, the distribution of hTERT is regulated by posttranslational modifications. Indeed, Src kinase has been shown to control the export of hTERT from the nucleus into the cytoplasm upon oxidative stress $[10,16]$. Dephosphorylation of hTERT by the phosphatase Shp-2 reversed this process [28]. Increased localization of hTERT to mitochondria upon oxidative stress was also reported [10]. 
High levels of mitochondrial hTERT were observed within hours of exposure to hydrogen peroxide $\left(\mathrm{H}_{2} \mathrm{O}_{2}\right)$ but several days were required in the case of hyperoxia. Although it took weeks, this redistribution of hTERT under hyperoxia was found to be a reversible process [10]. Together these data suggest that the intracellular redistribution of hTERT under oxidative stress likely relies on de novo protein synthesis but the current data do not exclude protein relocalization. Carrying out similar experiments in the presence of cycloheximide will help clarify this issue.

Büchner et al. [12] recently suggested that Src kinase also controls mitochondrial levels of hTERT upon oxidative stress similar to what has been shown for nuclear hTERT. The authors found that while levels of WT hTERT decreased in mitochondria upon $\mathrm{H}_{2} \mathrm{O}_{2}$ exposure, no changes occurred in a mutant version of hTERT (Y707F) that is not phosphorylated by Src kinase. They concluded that oxidative stress resulted in the Src-dependent mitochondrial downregulation of hTERT [12].

While it is possible that Src is involved in regulating nuclear and mitochondrial levels of hTERT under oxidative stress, the above data should be taken with some caution. The conclusions were based on targeting WT and Y707F hTERT to mitochondria by cloning it into a mitochondrial shooter vector, which essentially adds another MTS to the $\mathrm{N}$-terminus of hTERT [11]. However, no data showing the degree of mitochondrial localization of this construct was provided, which is required for proper interpretation of the data since levels of hTERT were assayed in total lysates prior to and after $\mathrm{H}_{2} \mathrm{O}_{2}$ treatment [12]. If under oxidative stress TERT indeed relocalizes to mitochondria, lack of complete mitochondrial localization of mitoTERT may skew interpretation of the results. Assaying levels of mitoTERT in isolated mitochondria under the same experimental conditions [11] could easily address this concern.

Regardless of the role of Src, the downregulation of mitochondrial hTERT could also stem from increased degradation of hTERT, decreased affinity of the mutant protein for the mitochondrial import machinery, or promotion of protein folding, which would compete for translocation [23-26]. Clearly, additional studies are required to better understand how the intracellular distribution and mitochondrial localization of hTERT are regulated, and whether the other stimuli reported to induce the subcellular shuttling of hTERT also influence the balance between mitochondrial and nuclear localization of the protein.

\section{4. hTERT and Its Role in mtDNA Metabolism}

Direct proof that TERT works in mtDNA metabolism is still missing but the ability of hTERT to modulate mtDNA damage induced by various agents, coupled with the recent demonstration that hTERT binds to mtDNA, strongly supports such a function. In this section we review evidence that mitochondrial hTERT promotes, protects, or does not interfere with mtDNA damage induced by different types of genotoxins. Taken together, the data indicate that the modulation of mtDNA damage caused by TERT is genotoxin-dependent and likely related to the type of lesion present on the mtDNA. The exact mechanism(s) through which hTERT modulates mtDNA damage has yet to be elucidated. We also discuss findings about the interaction of hTERT with the organellar DNA. However, we do not touch on the role of hTERT in stress-mediated cell death as this has been recently reviewed [18].

\subsection{Mitochondrial hTERT Modulates Oxidative Damage} on the mtDNA. As reviewed above, oxidative stress alters hTERT levels in mitochondria. Why under such conditions mitochondrial hTERT levels fluctuate is not clear but the available data suggest that it may respond to oxidative damage to mtDNA. Major controversy is observed regarding modulation of oxidative stress-mediated mtDNA damage, with evidence demonstrating that hTERT either exacerbates or protects against this type of insult $[7,8,10]$. The reasons for the opposing results are still unclear but they could reflect differences in the cellular backgrounds, in antioxidant defenses, and/or in the dose/length of the stress (see below).

We were the first to show that hTERT modulates mtDNA damage caused by $\mathrm{H}_{2} \mathrm{O}_{2}(200 \mu \mathrm{M}$ for either 15 minutes or 1 hour). Specifically, we demonstrated that cells expressing WT hTERT were more sensitive to $\mathrm{H}_{2} \mathrm{O}_{2}$-mediated mtDNA damage than the telomerase negative controls. Using 7 different cell lines expressing ectopic or endogenous hTERT and the respective non-hTERT isogenic controls, we showed that levels of mtDNA damage were significantly increased by the presence of the WT protein [7]. The promotion of damage was specific for hTERT because when hTERT was cloned flanked by lox $p$ and excised using cre recombinase the effects were reversed [8]. All cells showed similar kinetics of $\mathrm{H}_{2} \mathrm{O}_{2}$ breakdown; however, we detected an increase in bioavailable iron in cells overexpressing hTERT [7]. Iron can drive Fenton chemistry and more iron could increase the levels of hydroxyl radical generation, potentially explaining our results [7].

Subsequently, we established that the promotion of $\mathrm{H}_{2} \mathrm{O}_{2}$-induced mtDNA damage was strictly dependent on the presence of hTERT in mitochondria [8]. Stable expression of a nonmitochondrial mutant (R3E/R6EhTERT) completely abolished the promotion of mtDNA damage caused by $200 \mu \mathrm{M} \mathrm{H}_{2} \mathrm{O}_{2}$, leading to levels of lesions that were either not different from or even below their nonhTERT counterparts. Similar findings were observed using a catalytically inactive hTERT mutant, indicating that the promotion of mtDNA damage relied on reverse transcriptase activity. These results were obtained in different cellular backgrounds, demonstrating that the effects of mitochondrial hTERT were general and did not rely on a particular cell type. Together, these data led to the conclusion that telomerase must be active and in mitochondria to promote $\mathrm{H}_{2} \mathrm{O}_{2}$-induced mtDNA damage $[7,8]$.

Interestingly, work by Ahmed et al. in 2008 contradicts these findings. Using MRC-5 and MRC-5 stably expressing hTERT, they showed less damage in cells expressing exogenous hTERT when compared to the non-hTERT counterpart after treatment with $500 \mu \mathrm{M} \mathrm{H}_{2} \mathrm{O}_{2}$ for 3 hours. A similar trend was observed for cells put under hyperoxia conditions ( $40 \%$ oxygen) for up to 70 days. While levels of 
hyperoxia-induced mtDNA damage plateaued in MRC-5 cells from days 20 to 70, in MRC-5 hTERT the mtDNA damage was entirely eliminated after day 40 , when hTERT was solely mitochondrial localized [10]. These data suggest that (i) mtDNA repair was either not operating or was overwhelmed in MRC- 5 cells by the (likely) constant levels of damage induced by hyperoxia and (ii) that repair or turnover of hyperoxia-induced mtDNA damage was modulated by hTERT but only when the protein was in mitochondria. It is worth noting that the protection afforded by hTERT in this study could result from upregulation of antioxidant defenses since an increase in at least one antioxidant enzyme was detected in MRC-5 hTERT by gene expression profiles [10].

4.2. Effects of hTERT on mtDNA Damage Induced by Other Genotoxins. Methyl methanesulfonate (MMS) is a methylating agent that preferentially alkylates the $\mathrm{N}-7$ position of guanine. This lesion is converted into an abasic site and transiently into a single DNA strand break (SSB, [33]). Such lesions are repaired on the mitochondrial genome through the base excision repair pathway (BER, [34]). When hTERT overexpressing cells and their non-hTERT isogenic counterparts were exposed to MMS, no differences in the levels of either nuclear or mtDNA damage were observed. On the contrary, the trend pointed to a decrease in damage in both genomes in the presence of WT hTERT, which did not reach statistical significance [8].

As observed with $\mathrm{H}_{2} \mathrm{O}_{2}$, mtDNA damage induced by etoposide was promoted by hTERT. Indeed, a slight but significant enhancement in mtDNA damage was observed in NHF hTERT cells exposed to $1 \mu \mathrm{M}$ etoposide, which was not observed in NHF controls [8]. Etoposide, like $\mathrm{H}_{2} \mathrm{O}_{2}$, not only causes DNA double-strand breaks (DSBs) but induces oxidative stress as well [35]. Note that the majority of DNA breaks provoked by $\mathrm{H}_{2} \mathrm{O}_{2}$ are SSBs. In mitochondria, oxidized DNA and SSBs are repaired by BER whereas to date no system to correct DSBs has been identified in the mammalian organelle [34].

Mitochondrial hTERT was also proposed to protect mtDNA against UV damage. Using in vitro assays it was shown that preincubation of isolated DNA with in vitro translated hTERT protected the DNA against UV exposure. To show that this effect was physiologically relevant and involved the mitochondrial function of TERT, lung fibroblasts from WT and TERT knockout (KO) animals were exposed to increasing doses of UV and mitochondrial function accessed based on the MTT assay. Significantly less MTT conversion was observed in the KO cells with increasing doses of UV, leading to the conclusion that mitochondrial TERT protects mtDNA in vitro and mitochondrial function in vivo from UV damage [11]. Since nuclear TERT is also absent in the $\mathrm{KO}$ animals, further studies are required to define whether the protection afforded by TERT against UV damage indeed relies on its mitochondrial activity.

Likewise, expression of WT hTERT alleviated mtDNA depletion caused by low doses of ethidium bromide (EtBr) treatment. This rescue effect was more pronounced with expression of a mitochondrially targeted hTERT [11].
Potential mechanisms through which hTERT could be protecting the mtDNA from damage induced by UV and EtBr that cause, respectively, pyrimidine dimers and interand intrastrand crosslinks are unknown. Repair systems to remove these complex lesions have not been identified in mitochondria [34]. Based on the notion that mtDNA molecules containing nonrepairable lesions are targeted for degradation, one could speculate that hTERT may promote mtDNA turnover. Such activity is consistent with our previously proposed role for hTERT in mitochondrial quality control [8]. Other proposed that means through which TERT may modulate mtDNA damage are (i) modulation of BER, (ii) increase in intracellular bioavailable iron, (iii) changes in antioxidant defenses, and/or (iv) processing of mitochondrial DSBs. More studies are required to define the exact mechanism(s) through which TERT alters mtDNA damage and, importantly, what kinds of lesions are impacted by TERT.

4.3. hTERT Associates with mtDNA. If TERT was involved in mtDNA metabolism, one would predict that it binds mtDNA. Indeed, recent studies demonstrate that hTERT interacts with the mitochondrial genome. Using chromatin immunoprecipitations in cultured cells, Haendeler and colleagues demonstrated that hTERT interacts with mtDNA, consistent with its presence in the mitochondrial matrix. Surprising, however, was the fact that only two regions of the mtDNA were pulled down with hTERT: ND1 and ND2 of complex I [11]. Why only these two regions were identified as bound to TERT, which has been shown to have preference for telomeric DNA sequences [36], is not clear. While mtDNA does not have a telomeric structure, it does contain 22 telomeric-like repeats distributed across the two strands of the genome [7]. Such sequences could potentially be recognized by mitochondrial TERT and justify the preference for ND1 and ND2 binding. However, analysis of these gene sequences revealed no TTAGGG repeats (data not shown). Other potential explanations for hTERT association with mtDNA may relate to the oxidative state of the mitochondria during the experiments or the use of transient hTERT expression. Regardless, the binding of hTERT to the mitochondrial genome certainly strengthens its putative role in mtDNA metabolism. It will be interesting to define whether other regions of the mtDNA bind to TERT when the protein is stably expressed and, more importantly, whether such interaction changes upon genotoxic stress.

\section{TERT and Mitochondrial Biology}

MtDNA integrity is intimately related to mitochondrial function. Once mtDNA is damaged, expression of critical protein components of the electron transport chain (ETC) is decreased leading to increased reactive oxygen species (ROS) generation and decreased mitochondrial membrane potential $(\Delta \Psi \mathrm{m})$. Such events lead to a vicious cycle of mitochondrial damage, ultimately affecting mitochondrial import, calcium homeostasis, and ATP production among other processes [37-39]. Consistent with a role for TERT in mtDNA metabolism, recent work has shown that the absence 
of hTERT is associated with mitochondrial dysfunction. For instance, cells lacking hTERT showed higher mitochondrial ROS and decreased respiratory chain efficiency when compared to controls $[10,11,27]$. This section reviews evidence regarding the impact of hTERT on various mitochondrial parameters under basal conditions. Together the data reinforce the emerging role of hTERT in mitochondrial biology.

5.1. hTERT and ROS Production. Using mitoSOX and dihydrorhodamine (DHR) to detect mitochondrial superoxide $\left(\mathrm{O}_{2}{ }^{\bullet-}\right)$ and cellular peroxides (such as $\left.\mathrm{H}_{2} \mathrm{O}_{2}\right)$ it was shown that fluorescence from both dyes was decreased in MRC-5 hTERT cells when compared to MRC-5. These data indicate that the presence of hTERT was associated with decreased mitochondrial ROS production [10]. In agreement, overexpression of WT hTERT in Hek293 decreased levels of ROS when compared to empty-vector control or a catalytically inactive mutant [11]. In complementary experiments, siRNA knockdown of hTERT in HUVEC and Hek293 cells showed increased production of $\mathrm{O}_{2}{ }^{--}$and $\mathrm{H}_{2} \mathrm{O}_{2}$, confirming the notion that hTERT lowers the basal levels of ROS generation $[10,11]$.

Recently, we showed that expression of a mutant hTERT (NES-hTERT) that is unable to shuttle between subcellular compartments exhibited decreased mtDNA integrity and increased mitochondrial ROS [27]. These data are consistent with the above-mentioned work $[10,11]$ and suggest that nonnuclear hTERT is associated with improved mitochondrial function. However, our data did not rule out the possibility that the mitochondrial defects observed upon expression of NES-hTERT were associated with the senescent phenotype of the cells or to the defect in shuttling of hTERT itself [27]. It is important to note that these data were collected under nonstress conditions and therefore do not contradict our previous findings which were obtained under $\mathrm{H}_{2} \mathrm{O}_{2}$ exposure $[7,8]$. Although it would be interesting to submit NES-hTERT-expressing cells to oxidative stress, the data obtained will need to be interpreted with caution given the high degree of telomere damage and premature senescence triggered by expression of this mutant hTERT [27].

5.2. hTERT and Respiratory Chain Activity. The energy released by the transport of electrons through the ETC also provides protons used to establish an electrochemical gradient across the inner mitochondrial membrane [39]. Using JC-1 and confocal microscopy, it was shown that MRC-5 hTERT cells had significantly higher $\Delta \Psi \mathrm{m}$ at normoxic conditions when compared to its isogenic non-hTERT control [10]. However, this seemed in apparent contradiction with the lower levels of ROS in these cells given there is more chance of ROS generation when the $\Delta \Psi \mathrm{m}$ is high $[40,41]$. The authors suggested that the mitochondria of hTERT cells are tightly coupled, which explains the low ROS levels [10]. Indeed we recently showed that cells expressing WT hTERT have highly coupled mitochondria [27].

The activity of the ETC was directly evaluated using cells expressing either WT hTERT or a catalytically inactive mutant. Overall succinate-dependent respiratory function was $30 \%$ lower in the mutant while complex I activity was higher in cells carrying the WT protein. The increased respiratory capacity detected in cultured cells was confirmed in vivo. Respiratory function in intact heart and liver mitochondria isolated from WT and KO TERT animals was analyzed. Complex I activity was lower in the heart of the $\mathrm{KO}$ animals; however, no changes were detected in liver mitochondria. These data led to the hypothesis that in vivo the impact of mitochondrial TERT may be more prominent in cells that have high-energy requirements and less regenerative capacity [11].

The improvement in complex I function was attributed to the direct binding of hTERT to ND1 and ND2, which could potentially increase expression of these proteins [11]. Recent data in yeast suggest that enhanced mitochondrial respiration may be mediated by increased number of oxidative phosphorylation (OXPHOS) complexes per organelle as opposed to increasing the total number of mitochondria per cell [42]. It will be interesting to test whether this is the case in mammalian cells and, if so, which OXPHOS complexes are modulated by hTERT in mitochondria.

Two TERT KO mouse models have been generated, and no gross defects have been observed in early generations $[43,44]$, inconsistent with a major role for TERT in mtDNA metabolism. It is important to note that results observed with cells grown in culture were mild, and it is possible that subtle differences have been overlooked when not specifically investigating mitochondrial function in these animals. Alternatively, it is feasible that compensatory mechanisms were activated in the $\mathrm{KO}$ animals. Interestingly, late generation mTERT KO animals had accelerated infertility, which was initially ascribed to the short telomeres present in the animals. However, mice heterozygous for TERT, while having very short telomeres, did not show the same phenotype, leading the authors to conclude that the dosage of TERT was important for genomic stability [45]. It is known that proper mitochondrial function plays an important role in fertility [46]. In light of the latest findings, it is tempting to speculate that the impact of the lack of mitochondrial TERT contributed to the infertility previously observed in the $\mathrm{KO}$ animals. Certainly, it seems worth revisiting the mouse models available to address whether mitochondrial abnormalities are present in these animals. Of particular interest will be to measure mitochondrial function in the $\mathrm{KO}$ animals under conditions that require a high-energy demand. Of note, TERT KO animals have been submitted to physical exercise, and an important role for telomerase has been ascertained in the heart and in aortic endothelial cells $[47,48]$. However, no direct assessment of mitochondrial function such as cytochrome c oxidase activity or ATP production was conducted nor was a connection to mitochondrial biology or to telomerase function in mitochondria established $[47,48]$, all worth addressing in future studies.

\section{Concluding Remarks}

Mitochondrial function is key to cellular homeostasis, and maintenance of mitochondrial quality has major impact in health and disease. A role for TERT in mitochondria is clearly 
emerging although its exact mechanism of action remains largely unknown. Years of work done in vitro and in vivo have established telomerase as a main player in aging and cancer primarily due to its action upon the telomeres. The mitochondrial localization and function of TERT bring yet another layer of complexity to the already intricate scenario of telomerase multitasking in the cell. More experiments are certainly required to differentiate the mitochondrial effects of TERT as dependent on its nuclear versus mitochondrial localization and to establish the biological significance of TERT in mitochondria in vivo. The next years will be very exciting for both the mitochondrial and telomerase fields as more details about the function of TERT in the organelle are unveiled. Such studies will allow for a better understanding of the overall role of telomerase in cellular function and will lay foundation for new therapeutic approaches to aging, ageand mitochondrial-related diseases, and cancer.

\section{Acknowledgments}

The authors thank Dr. Patricia L. Opresko from the Department of Environmental and Occupational Health and Dr. Hong Wang from the Department of Pharmacology and Chemical Biology of the University of Pittsburgh for critical review of the paper. They also thank the support from the Department of Defense through the Army Research Office (Award no. 56027LS to J. H. Santos).

\section{References}

[1] J. W. Shay and W. E. Wright, "Senescence and immortalization: role of telomeres and telomerase," Carcinogenesis, vol. 26, no. 5, pp. 867-874, 2005.

[2] A. Kilian, D. D. L. Bowtell, H. E. Abud et al., "Isolation of a candidate human telomerase catalytic subunit gene, which reveals complex splicing patterns in different cell types," Human Molecular Genetics, vol. 6, no. 12, pp. 2011-2019, 1997.

[3] K. Masutomi, E. Y. Yu, S. Khurts et al., "Telomerase maintains telomere structure in normal human cells," Cell, vol. 114, no. 2, pp. 241-253, 2003.

[4] K. Masutomi, R. Possemato, J. M. Y. Wong et al., "The telomerase reverse transcriptase regulates chromatin state and DNA damage responses," Proceedings of the National Academy of Sciences of the United States of America, vol. 102, no. 23, pp. 8222-8227, 2005.

[5] J.-I. Park, A. S. Venteicher, J. Y. Hong et al., "Telomerase modulates Wnt signalling by association with target gene chromatin," Nature, vol. 460, no. 7251, pp. 66-72, 2009.

[6] Y. Maida, M. Yasukawa, M. Furuuchi et al., "An RNAdependent RNA polymerase formed by TERT and the RMRP RNA," Nature, vol. 461, no. 7261, pp. 230-235, 2009.

[7] J. H. Santos, J. N. Meyer, M. Skorvaga, L. A. Annab, and B. Van Houten, "Mitochondrial hTERT exacerbates free-radicalmediated mtDNA damage," Aging Cell, vol. 3, no. 6, pp. 399$411,2004$.

[8] J. H. Santos, J. N. Meyer, and B. Van Houten, "Mitochondrial localization of telomerase as a determinant for hydrogen peroxide-induced mitochondrial DNA damage and apoptosis," Human Molecular Genetics, vol. 15, no. 11, pp. 1757-1768, 2006.
[9] D. Del Bufalo, A. Rizzo, D. Trisciuoglio et al., "Involvement of hTERT in apoptosis induced by interference with Bcl-2 expression and function," Cell Death and Differentiation, vol. 12, no. 11, pp. 1429-1438, 2005.

[10] S. Ahmed, J. F. Passos, M. J. Birket et al., "Telomerase does not counteract telomere shortening but protects mitochondrial function under oxidative stress," Journal of Cell Science, vol. 121, no. 7, pp. 1046-1053, 2008.

[11] J. Haendeler, S. Dröse, N. Büchner et al., "Mitochondrial telomerase reverse transcriptase binds to and protects mitochondrial DNA and function from damage," Arteriosclerosis, Thrombosis, and Vascular Biology, vol. 29, no. 6, pp. 929-935, 2009.

[12] N. Büchner, T. -C. Zschauer, M. Lukosz, J. Altschmied, and J. Haendeler, "Downregulation of mitochondrial telomerase reverse transcriptase induced by $\mathrm{H}_{2} \mathrm{O}_{2}$ is Src kinase dependent," Experimental Gerontology, vol. 45, no. 7-8, pp. 558-562, 2010.

[13] G. B. Morin, "The human telomere terminal transferase enzyme is a ribonucleoprotein that synthesizes TTAGGG repeats," Cell, vol. 59, no. 3, pp. 521-529, 1989.

[14] H. Seimiya, H. Sawada, Y. Muramatsu et al., "Involvement of 14-3-3 proteins in nuclear localization of telomerase," EMBO Journal, vol. 19, no. 11, pp. 2652-2661, 2000.

[15] B. N. Armbruster, S. S. R. Banik, C. Guo, A. C. Smith, and C. M. Counter, "N-terminal domains of the human telomerase catalytic subunit required for enzyme activity in vivo," Molecular and Cellular Biology, vol. 21, no. 22, pp. 77757786, 2001.

[16] J. Haendeler, J. Hoffmann, R. P. Brandes, A. M. Zeiher, and S. Dimmeler, "Hydrogen peroxide triggers nuclear export of telomerase reverse transcriptase via Src kinase familydependent phosphorylation of tyrosine 707," Molecular and Cellular Biology, vol. 23, no. 13, pp. 4598-4610, 2003.

[17] J. Haendeler, J. Hoffmann, J. F. Diehl et al., "Antioxidants inhibit nuclear export of telomerase reverse transcriptase and delay replicative senescence of endothelial cells," Circulation Research, vol. 94, no. 6, pp. 768-775, 2004.

[18] G. Saretzki, "Telomerase, mitochondria and oxidative stress," Experimental Gerontology, vol. 44, no. 8, pp. 485-492, 2009.

[19] C. Belgiovine, I. Chiodi, and C. Mondello, "Telomerase: cellular immortalization and neoplastic transformation. Multiple functions of a multifaceted complex," Cytogenetic and Genome Research, vol. 122, no. 3-4, pp. 255-262, 2009.

[20] F. M. Bollmann, "The many faces of telomerase: emerging extratelomeric effects," BioEssays, vol. 30, no. 8, pp. 728-732, 2008.

[21] Y. Cong and J. W. Shay, "Actions of human telomerase beyond telomeres," Cell Research, vol. 18, no. 7, pp. 725-732, 2008.

[22] S. B. Cohen, M. E. Graham, G. O. Lovrecz, N. Bache, P. J. Robinson, and R. R. Reddel, "Protein composition of catalytically active human telomerase from immortal cells," Science, vol. 315, no. 5820, pp. 1850-1853, 2007.

[23] N. Regev-Rudzki and O. Pines, "Eclipsed distribution: a phenomenon of dual targeting of protein and its significance," BioEssays, vol. 29, no. 8, pp. 772-782, 2007.

[24] A. Naamati, N. Regev-Rudzki, S. Galperin, R. Lill, and O. Pines, "Dual targeting of Nfs1 and discovery of its novel processing enzyme, Icp55," Journal of Biological Chemistry, vol. 284, no. 44, pp. 30200-30208, 2009.

[25] N. Regev-Rudzki, O. Yogev, and O. Pines, “The mitochondrial targeting sequence tilts the balance between mitochondrial and cytosolic dual localization," Journal of Cell Science, vol. 121, no. 14, pp. 2423-2431, 2008. 
[26] O. Yogev and O. Pines, "Dual targeting of mitochondrial proteins: mechanism, regulation and function," Biochimica et Biophysica Acta. In press.

[27] O. A. Kovalenko, M. J. Caron, P. Ulema et al., "A mutant telomerase defective in nuclear-cytoplasmic shuttling fails to immortalize cells and is associated with mitochondrial dysfunction," Aging Cell, vol. 9, no. 2, pp. 203-219, 2010.

[28] S. Jakob, P. Schroeder, M. Lukosz et al., "Nuclear protein tyrosine phosphatase Shp-2 is one important negative regulator of nuclear export of telomerase reverse transcriptase," Journal of Biological Chemistry, vol. 283, no. 48, pp. 33155-33161, 2008.

[29] M. Akiyama, T. Hideshima, T. Hayashi et al., "Nuclear factor$\kappa \mathrm{B}$ p65 mediates tumor necrosis factor $\alpha$-induced nuclear translocation of telomerase reverse transcriptase protein," Cancer Research, vol. 63, no. 1, pp. 18-21, 2003.

[30] K. Liu, R. J. Hodes, and N.-P. Weng, "Cutting edge: telomerase activation in human $\mathrm{T}$ lymphocytes does not require increase in telomerase reverse transcriptase (hTERT) protein but is associated with hTERT phosphorylation and nuclear translocation," Journal of Immunology, vol. 166, no. 8, pp. 4826-4830, 2001.

[31] A. Kimura, M. Ohmichi, J. Kawagoe et al., "Induction of hTERT expression and phosphorylation by estrogen via Akt cascade in human ovarian cancer cell lines," Oncogene, vol. 23, no. 26, pp. 4505-4515, 2004.

[32] R. Ram, O. Uziel, O. Eldan et al., "Ionizing radiation up-regulates telomerase activity in cancer cell lines by post-translational mechanism via ras/phosphatidylinositol 3kinase/Akt pathway," Clinical Cancer Research, vol. 15, no. 3, pp. 914-923, 2009.

[33] S. H. Wilson, "Mammalian base excision repair and DNA polymerase beta," Mutation Research, vol. 407, no. 3, pp. 203 215, 1998.

[34] B. S. Mandavilli, J. H. Santos, and B. Van Houten, "Mitochondrial DNA repair and aging," Mutation Research, vol. 509, no. 1-2, pp. 127-151, 2002.

[35] P. Meresse, E. Dechaux, C. Monneret, and E. Bertounesque, "Etoposide: discovery and medicinal chemistry," Current Medicinal Chemistry, vol. 11, no. 18, pp. 2443-2466, 2004.

[36] D. C. F. Sealey, L. Zheng, M. A. S. Taboski, J. Cruickshank, M. Ikura, and L. A. Harrington, "The N-terminus of hTERT contains a DNA-binding domain and is required for telomerase activity and cellular immortalization," Nucleic Acids Research, vol. 38, no. 6, pp. 2019-2035, 2009.

[37] B. Van Houten, V. Woshner, and J. H. Santos, "Role of mitochondrial DNA in toxic responses to oxidative stress," DNA Repair, vol. 5, no. 2, pp. 145-152, 2006.

[38] D. C. Wallace, "Mitochondria as chi," Genetics, vol. 179, no. 2, pp. 727-735, 2008.

[39] D. C. Wallace and W. Fan, "The pathophysiology of mitochondrial disease as modeled in the mouse," Genes and Development, vol. 23, no. 15, pp. 1714-1736, 2009.

[40] R. S. Balaban, S. Nemoto, and T. Finkel, "Mitochondria, oxidants, and aging," Cell, vol. 120, no. 4, pp. 483-495, 2005.

[41] M. P. Murphy, "How mitochondria produce reactive oxygen species," Biochemical Journal, vol. 417, no. 1, pp. 1-13, 2009.

[42] G. S. Shadel and Y. Pan, "Multi-faceted regulation of mitochondria by TOR," Cell Cycle, vol. 8, no. 14, p. 2143, 2009.

[43] X. Yuan, S. Ishibashi, S. Hatakeyama et al., "Presence of telomeric G-strand tails in the telomerase catalytic subunit TERT knockout mice," Genes to Cells, vol. 4, no. 10, pp. 563$572,1999$.
[44] Y. Liu, B. E. Snow, M. P. Hande et al., "The telomerase reverse transcriptase is limiting and necessary for telomerase function in vivo," Current Biology, vol. 10, no. 22, pp. 1459-1462, 2000.

[45] N. Erdmann, Y. Liu, and L. Harrington, "Distinct dosage requirements for the maintenance of long and short telomeres in mTert heterozygous mice," Proceedings of the National Academy of Sciences of the United States of America, vol. 101, no. 16, pp. 6080-6085, 2004.

[46] M. Terzioglu and N.-G. Larsson, "Mitochondrial dysfunction in mammalian ageing," Novartis Foundation Symposium, vol. 287, pp. 197-208, 2007.

[47] C. Werner, M. Hanhoun, T. Widmann et al., "Effects of physical exercise on myocardial telomere-regulating proteins, survival pathways, and apoptosis," Journal of the American College of Cardiology, vol. 52, no. 6, pp. 470-482, 2008.

[48] C. Werner, T. Fürster, T. Widmann et al., "Physical exercise prevents cellular senescence in circulating leukocytes and in the vessel wall," Circulation, vol. 120, no. 24, pp. 2438-2447, 2009. 

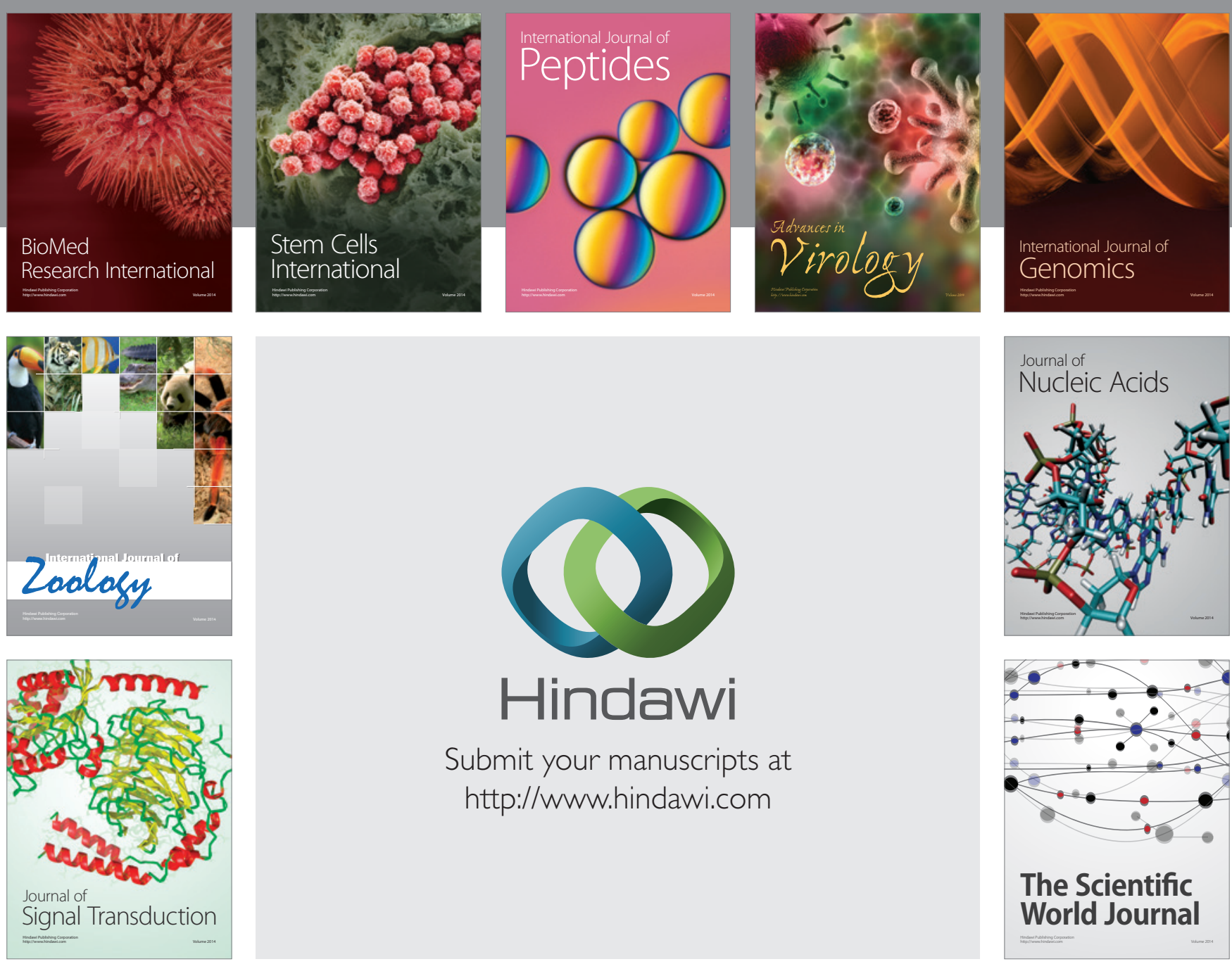

Submit your manuscripts at

http://www.hindawi.com
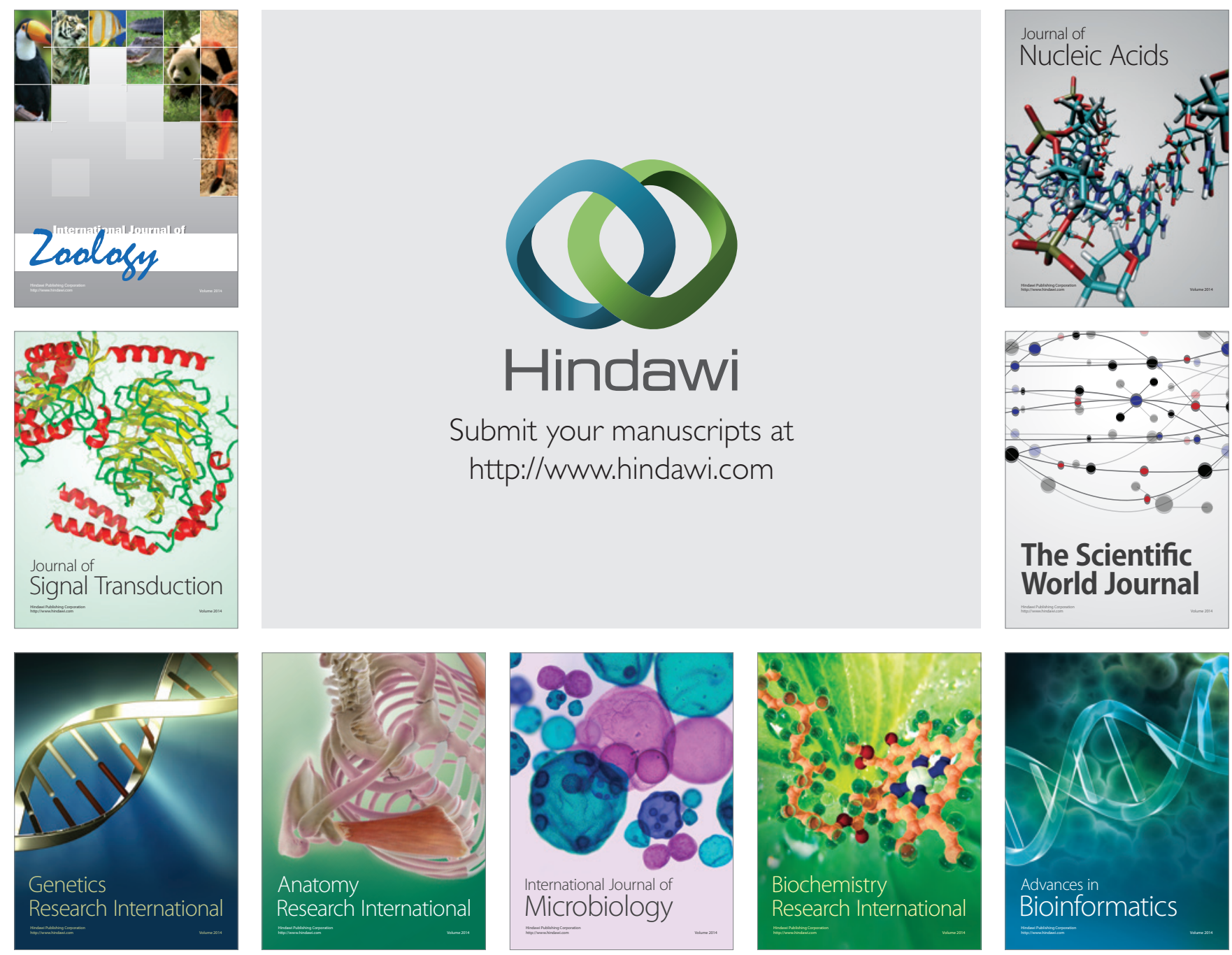

The Scientific World Journal
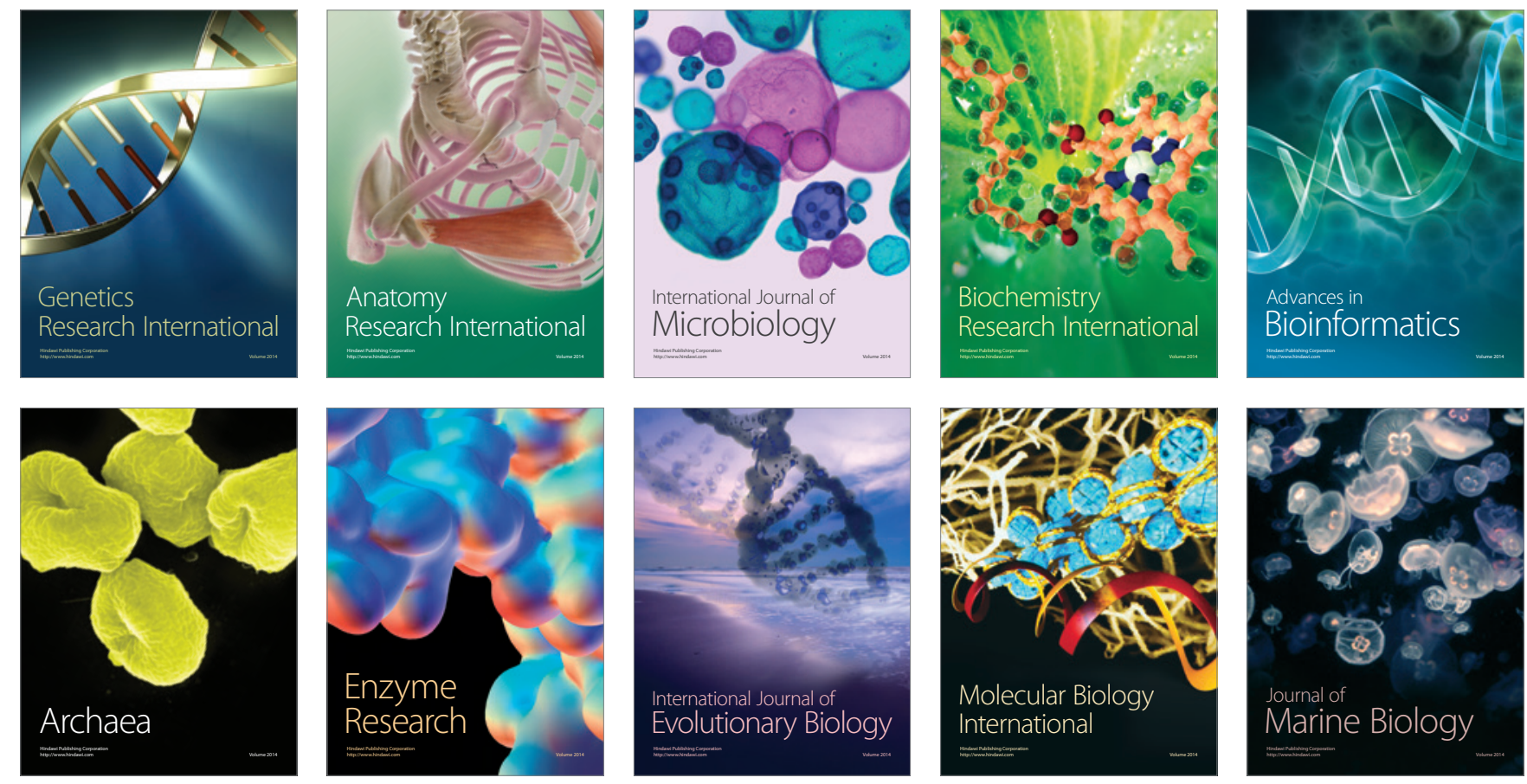